\title{
Design and Modelisation of a Straylight Facility for Space Optical Instrument
}

\author{
E. Mazy ${ }^{\mathrm{a}}$, Y. Stockman ${ }^{\mathrm{b}}$, M.L. Hellin ${ }^{\mathrm{a}}$ \\ ${ }^{\mathrm{a}}$ Centre Spatial of Liege, Avenue du Pre-Aily, 4030 Angleur, Belgium
}

\begin{abstract}
In the framework of instrument calibration, straylight issues are a critical aspect that can deteriorate the optical performances of instrument. To cope with this, a new facility is designed dedicated for in-field and far field straylight characterization: up to $10^{-8}$ for in-field and up to $10^{-10}$ for far field straylight in the visible to NIR spectral ranges. Moreover, from previous straylight test performed at CSL, vacuum conditions are needed for reaching the $10^{-10}$ rejection requirement mainly to avoid air/dust diffusion. The major constrains are to design a straylight facility either for in-field and out-field straylight measurements. That requires high dynamic range at source level and a high radiance point source allowing small diverging collimated beam. Moreover, the straylight facility has to be implemented into a limited envelope and has to be built with vacuum compatible materials and black coating. As checking the facility performance requires an instrument better than the facility itself, that is no easy to find, so that the performances have been estimated through a modelisation into a non sequential optical software. This modelisation is based on CAD importation of mechanical design, on BRDF characteristics of black coating and on statistical averaging of ray tracing at instrument entrance.
\end{abstract}

Keywords: Straylight facility, straylight modelisation, straylight design, BRDF, black coating scattering

\section{INTRODUCTION}

The Centre Spatial de Liege (CSL) has been involved since twenty years in the characterization and evaluation of stray light for space instruments (ref [1] to [5]). Today CSL is developing a new stray light facility for the stray light characterization of small earth observation satellites. Stray light issues are tackled in different ways. For large payloads deep stray light analysis is carried out, tests at subsystem are performed or partial illuminations are also envisaged. For small EO satellite, it is possible to perform an end to end test to evaluate the stray light characteristic of the instrument.

\section{TEST FACILITY REQUIREMENTS}

For an optical system, the stray light contributions may be summed as In-field Stray light (IFS) and Out-of-field Stray light (OFS). The major stray light contributions are:

- the effects of mirror microroughness,

- the effects of dust or defects on the mirrors,

- the scattering induced by the aperture stop,

- the effects of ghosts,

- the stray light due to Sun and Moon, or any intense light source out of the FOV.

The facility will not be able to directly identify the sources of stray light listed here above, but will verify that the stray light contribution for along angles (taken in absolute values) larger than 10 arc degrees or for across angles larger than 25 arc degrees are negligible, and that the dominant contributor to stray light for in field is the pupil stop. The mirror microroughness, dust and defects contributions are indistinguishable in practice and bear a strong angular variation from the incident direction.

The test facility is designed for infield straylight characterization up to $10^{-8}$ as close as \pm 250 arcsec from the incident and for farfield straylight characterization up to $10^{-10}$ as close as $\pm 10^{\circ}$ from the incident. 


\section{TEST FACILITY CONCEPT DESCRIPTION}

\subsection{Stray light test facility overview}

The facility is in a clean room to eliminate undesired air particles scatter light sources and to allow to work with space hardware. To cope with this, the stray light facility is developed in a vacuum chamber. The baseline is to use the vacuum chamber closed, in such a way that the stray light level from the external environment is negligible.

Inside the chamber dedicated baffle design are studied to eliminate undesired light generated by the set up itself e.g backscattered light away from the instrument under test. This implies blackened shrouds (painted with MAP PU1) inside the facility.

The proposed illumination starts from a $400 \mathrm{~mm}$ off axis parabolic available at CSL. The off axis design suppresses the problem of stray light generated by the internal obstruction. To reach the infield straylight requirement, a dedicated source assembly is developed to avoid any stray light coming from the structure around the source pinhole as well as the fold mirror.

The payload is set on a GSE allowing remote control tip/tilt adjustment. The GSE consists in a rotation of $+/-180^{\circ}$ and tip/tilt from $-10+10^{\circ}$.

Dedicated attention is required on the selection of the light source to achieve the large required measurement dynamic range and to the stray light analysis. This is basically the only way to guarantee that there is no residual stray light coming from the facility itself.

A general design of the facility is presented in Figure 1. The facility consists in the following items:

1) the vacuum chamber

2) the collimator in an auxiliary chamber

3) the source pack

4) the rotating table

5) baffle design

Each of these subsystems will be described here after.

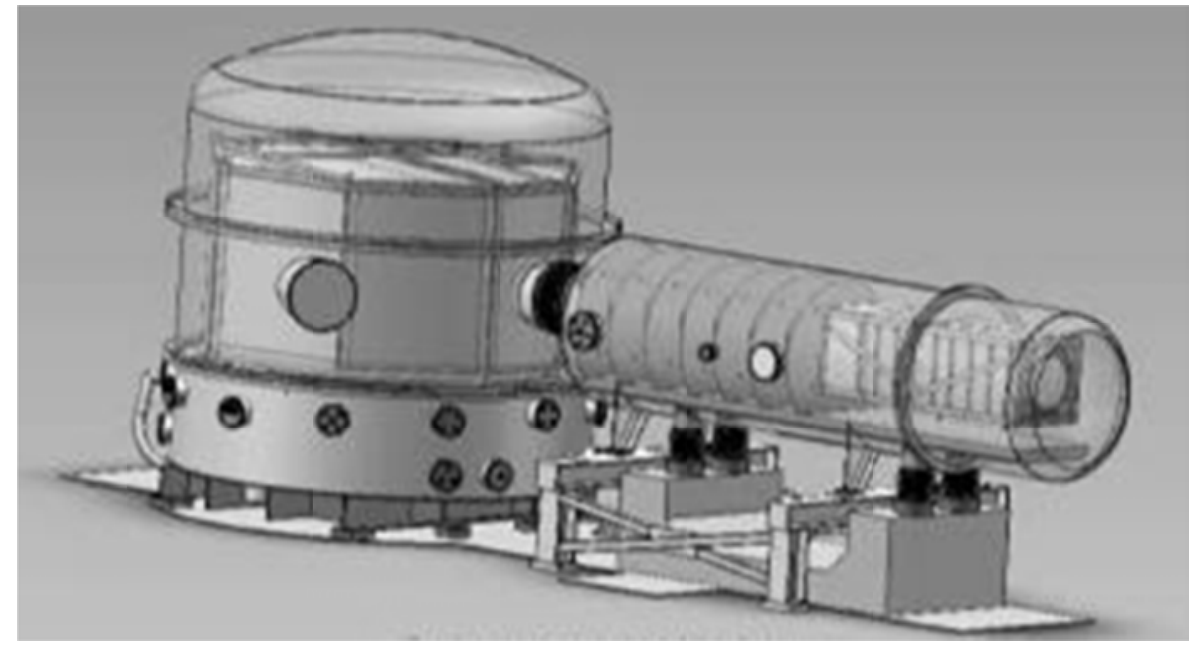

Figure 1. Stray ligth facility general overview

\subsection{The vacuum chamber}

The set up is implemented in FOCAL 3 facility. FOCAL 3 (acronym of Facility for Optical Calibration At Liège) is composed of two stainless steel vessels located in a class 10000 clean room (following US standard: FED-STD-209E). One main vertical cylindrical chamber of $3 \mathrm{~m}$ diameter and $2.8 \mathrm{~m}$ height and an auxiliary horizontal axis of $1.2 \mathrm{~m}$ 
diameter and $5 \mathrm{~m}$ length. The stray light test set-up is developed in a CL100 cleanliness. Two optical benches are installed respectively in the main chamber and in the auxiliary chamber. Both benches are put on the same seismic block which is actively controlled thanks to 5 air cushions. Focal 3 chamber will be used at air for infield measurements. Literature and CSL experience indicated that, up to $10^{-8}$, air scattering is not an issue. Primary pumping is foreseen when measurements down to $10^{-10}$ need to be achieved.

\subsection{The collimator}

The collimator consists in a $400 \mathrm{~mm}$ off-axis parabola with a $2 \mathrm{~m}$ focal length. It will be placed in the auxiliary chamber as far as possible from the tested payload (about $5 \mathrm{~m}$ ). A stainless steel truss supports the parabolic mirror cell and the focal plane. The trust is black painted with MAP PU1 and backed (see Figure 2).

\subsection{The collimator baffling}

The collimator baffling is divided in two parts. A first baffling of the truss (see Figure 2), that consists in a set of 4 black MAP PU1 Aluminum sheets to close the sides of the truss. These sheets have 10 additional vanes to avoid grazing incidence.

A second baffling (see Figure 2) is placed in the auxiliary chamber to hide the visibility of the metallic parts of the vacuum chamber with respect to the instrument. It consists of a set of 7 black MAP PU1 Aluminum sheets with an central aperture of $440 \mathrm{~mm}$.
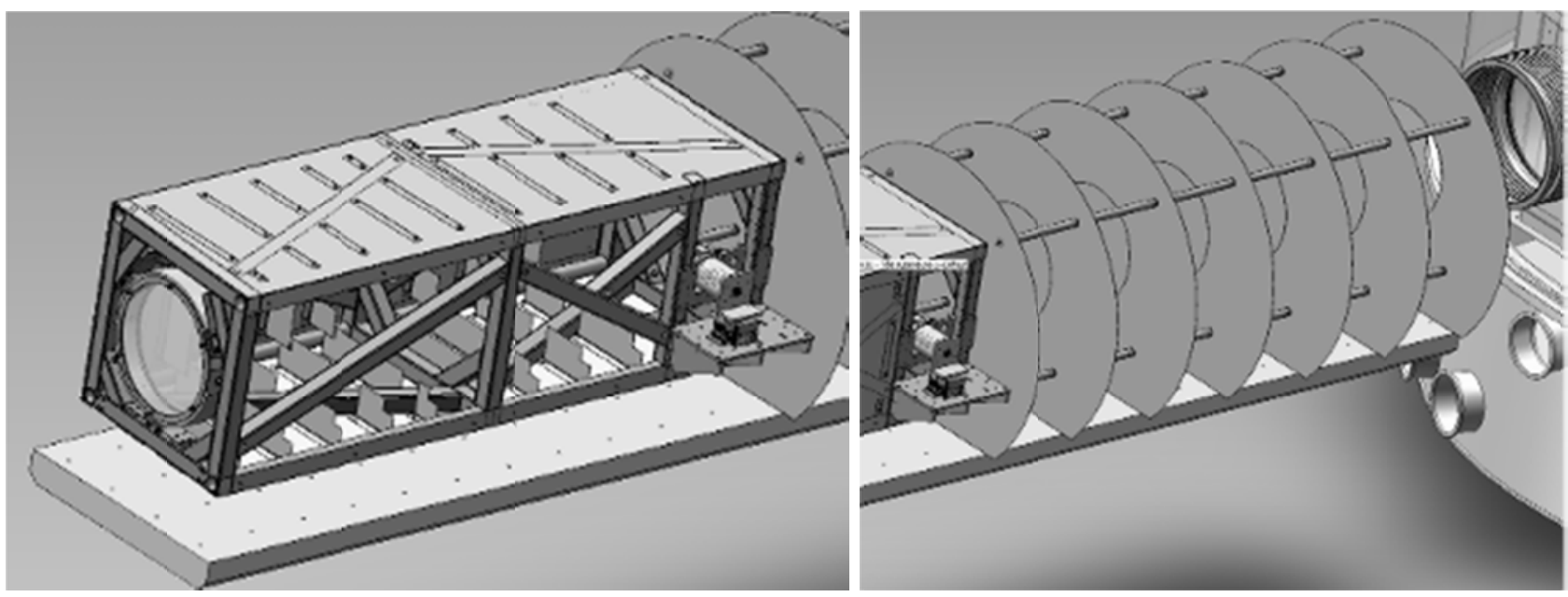

Figure 2. Collimator trust baffling (left) and auxiliary chamber baffling (right)

\subsection{The source pack}

The Source pack requires several items. In a first time an adequate baffling to avoid stray light in the FOV coming from the source itself. Secondly it necessitates a strong source since very low level of stray light needs to be detected. To fulfill this two sources are used, one plasma source with a $230 \mu \mathrm{m}$ core Optical Fiber (OF) for IFOV and one NIR $20 \mathrm{~W}$ laser diode with a $600 \mu \mathrm{m}$ core OF for OFOV measurements. To manage the great dynamic range an attenuation system is also implemented. This is controlled with monitoring detectors.

\subsection{Source baffling}

This baffling is a critical part of the set up for the infield stray light performances. The goal is to have a system able to illuminate only one single pixel of the instrument and to get a level lower than $10^{-8}$ in the neighbour pixels. It is required that this level of $10^{-8}$ is achieved after 250 arcsec. Since this is very difficult to measure, the requirements are demonstrated by analysis.

The source baffling has 2 main objectives. A first is to avoid any straylight coming from reflection of parts close to the source or backscattered to the source; a second is to limit the output beam $\mathrm{F}_{\text {number }}$ in order to avoid illumination of the parabolic mirror mount which is in direct view with the tested instrument. 
To minimize these stray light contributions, a source assembly has been specially designed based on a NG1 glass pyramid for infield stray light contributions, an aperture stop to limit the $F_{\text {number }}$ and a set of internal vanes to limit farfield straylight.

Two models of NG1 glass pyramid with respectively a $\Phi 300 \mu \mathrm{m}$ hole for the $\Phi 230 \mu \mathrm{m}$ optic fiber and a $\Phi 800 \mu \mathrm{m}$ hole for the $\Phi 600 \mu \mathrm{m}$ optic fiber are manufactured. The side of the pyramid are polished and tilted to minimized reflection and diffusion towards the aperture stop. To go from one pyramid to the other, the mechanical pyramids are designed to allow the replacement with an accuracy better than $50 \mu \mathrm{m}$. interface. Nevertheless, no motorized units will be used, so that to change the source, it will be requested to open the auxiliary chamber.

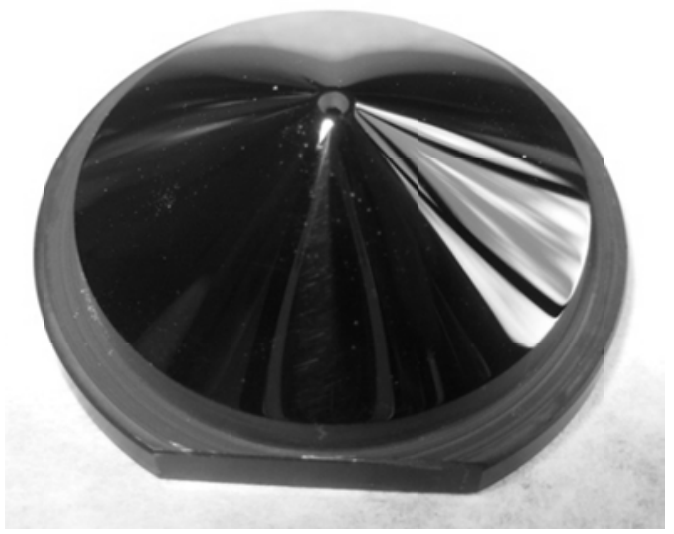

Figure 3: NG1 glass pyramid before optic fiber installation.

The aperture stop on both sides and all internal vanes are black coated with $A c k t a r{ }^{\circledR}$ vacuum black $\left(\mathrm{R}_{\text {hemispherical }}<1.6 \%\right)$ in order to reduce straylight in the farfield.

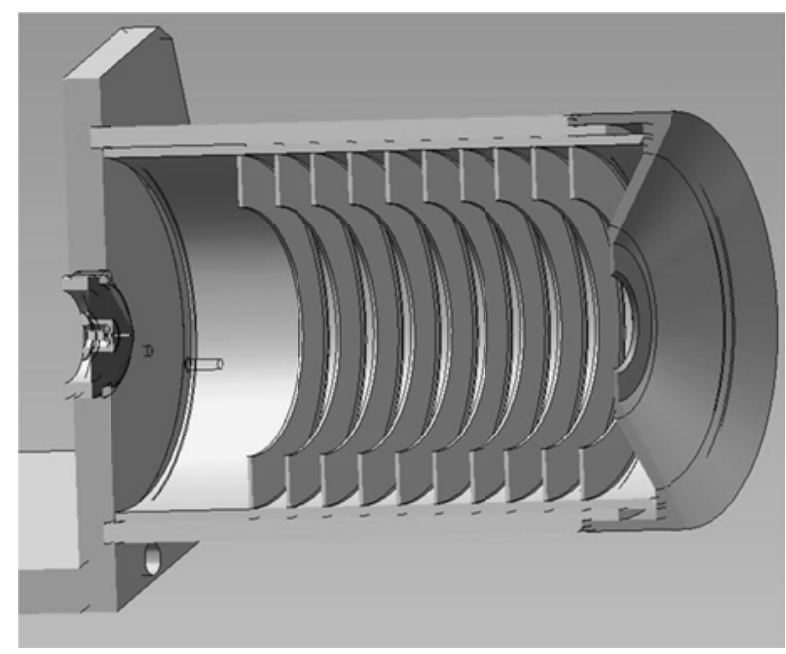

Figure 4. Light trap model

\subsection{Light sources}

The baseline is to use fiber coupled source with the highest radiance possible. For nearfield measurements, the proposed plasma source presents a spectral integrated stability of $0.018 \% 1 \sigma$ standard deviation. The fact that it is a fibered source allows to put all the heating parts and potential stray light source outside the chamber and to feed the focal plane only with a $230 \mu \mathrm{m}$ optical fiber. For the farfield measurements, a NIR $20 \mathrm{~W}$ laser diode pigtailed into a $600 \mu \mathrm{m}$ optic fiber is used. Both sources are linked to a coupling and attenuation system to allow attenuation of the source outside the chamber. It is realized with an achromatic afocal system (x1 magnification) into which Neutral Density Filter are introduced to attenuate the beam. 


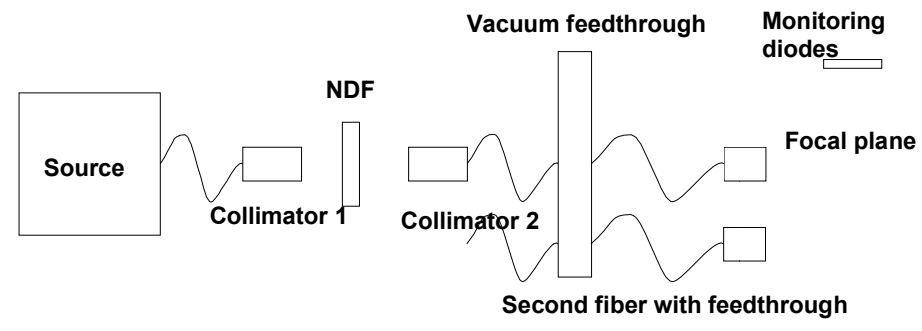

Figure 5. Source pack layout I/F

\subsection{Flux stability monitoring}

Since long integration time is required, the flux intensity will be monitored. This will be realized with 2 vacuum compatible detectors able to measure $10^{-4}$ beam intensity variation. Internal attenuation in one detector allow to fit with a $10^{-8}$ range. The required $10^{-10}$ range is reached by increasing the integration time on the instrument. The monitoring will be placed into the collimated beam and will collect a small part of the collimated beam.

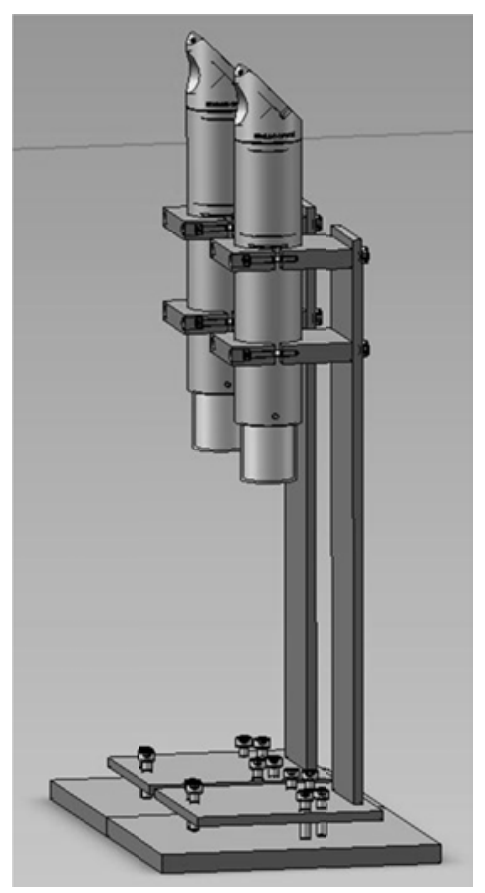

Figure 6. Monitoring assembly

\section{BAFFLE DESIGN}

One of the most critical parts in the design of the stray light facility is to get rid of all internal stray light coming from reflection of the wall of the chamber or retro reflection from the tested instrument. To limit this stray light, it is proposed to put the payload in a black box as large as possible: the chamber surrounded by black painted walls. Additionally a payload baffle in front of the entrance aperture will control the back reflection onto the instrument.

\subsection{Facility baffle design}

The instrument will be completely surrounded by a $\Phi 3 \mathrm{~m}$ diameter cylinder black painted with MAP PU1. The instrument is set on a MGSE on the rear of the black tent. A black painted Kapton foils make the link between the MGSE and the facility baffles, allowing MGSE rotation without interference. To reduce diffuse back reflection into the FOV of the instrument, a $1 \mathrm{~m}$ height band spread over $180^{\circ}$ is additionally overcoated with Acktar ${ }^{\circledR}$ Metal Velvet sheets $\left(\mathrm{R}_{\text {hemispherical }}<1 \%\right)$ 

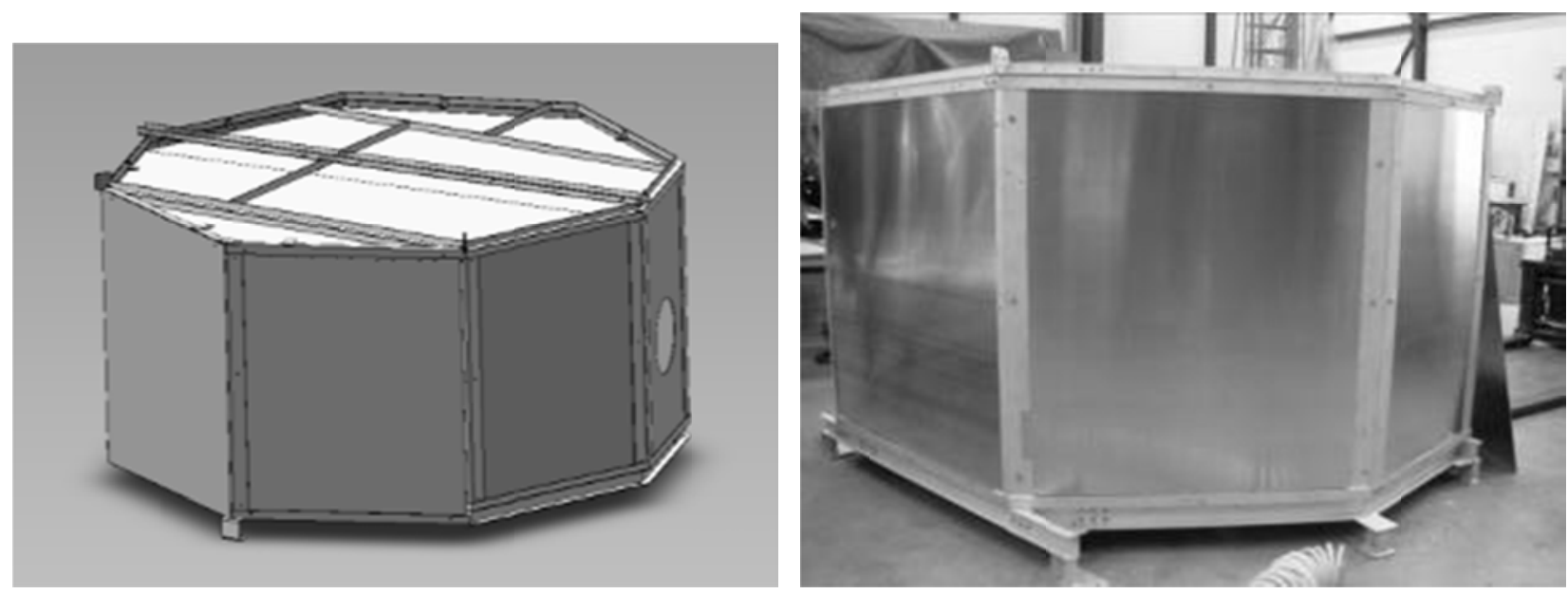

Figure 7. Facility baffling drawing and before paintingr

\subsection{Payload baffling}

In order to reduce impact of payload metallic part on the straylight measurement, a small baffle is put at the level of the entrance payload primary baffle to block any remaining light coming from the collimator outside the baffle aperture. Care is taken on this baffle since it received the full beam of the collimator and this baffle should retro reflect light as less as possible. Payload baffle will be black coated with Acktar ${ }^{\circledR}$ Metal Velvet sheets.

\section{MECHANICAL GOUND SUPPORT EQUIPMENT}

The MGSE is the same as the one developed for the PROBA V calibration ([6] and [7]). The rotating table is a XMM heritage that was used to calibrate the XMM Newton Mirror Modules. The table is able to support more than 1 ton. The angular range is $+/-200^{\circ}$, with a resolution lower than 10 arcsec. To achieve the along track angle, a tip/tilt table inherited from the Planck Primary reflector cryo characterization is used and is interfaced on the rotating table. The motors have a stroke of $200 \mathrm{~mm}$ allowing to achieve a range of $+/-13$ arcdegrees. The coders on the translation motor have a resolution of $2 \mu \mathrm{m}$, that will allow a tilt position with a resolution of 1 arcsec.

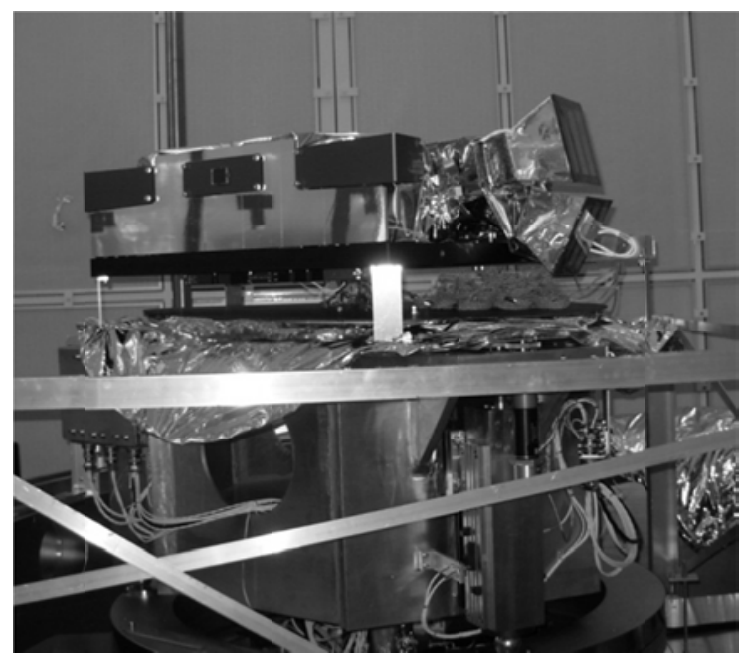

Figure 8. ProbaV onto the MGSE. 


\section{OGSE STRAYLIGHT PERFORMANCES}

\subsection{Radiometric budget}

With the selected source (i.e. plasma source), the achieved radiance levels at the output of the collimator are about 4000 $\mathrm{W} / \mathrm{m}^{2}$.sr. $\mu \mathrm{m}$, which is about 4 to 5 decades brighter that the L1 ground luminance level. The 3 to 4 additional decades can be accessed by changing the integration time and keeping a SNR lower than 10 (this is pending on the detector performance of the tested payload). So, in practice a level of $10^{8}$ can be achieved.

To achieve the $10^{10}$ level for farfield stray light measurement, the laser diode is used. With the $20 \mathrm{~W}$ laser diode, this level is easily achieved (up to $10^{12}$ is possible with the same assumptions as here above).

\subsection{Stray light simulation: model}

The stray light facility is modeled in FRED $^{\circledR}$ non sequential ray tracing software. The model considers the CAD files of the collimator, baffling systems and F3 optical benches. The tested instrument is summarized by its front panel. The detector is a $50 \times 200 \mathrm{~mm}^{2}$ rectangle corresponding to the entrance baffle size of the payload. This can be enlarged until its stays within a diameter of $300 \mathrm{~mm}$.
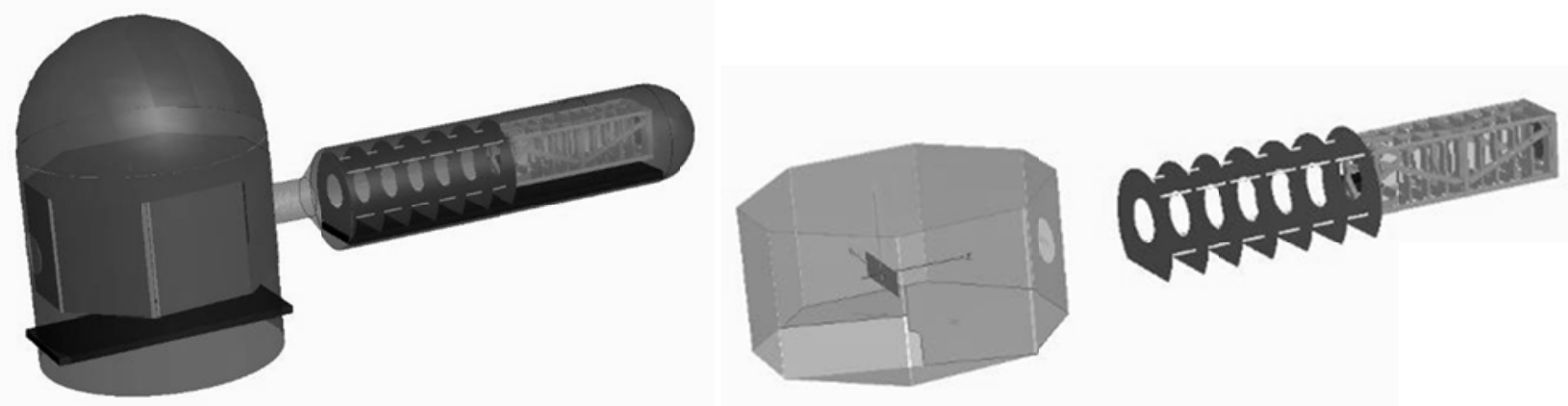

Figure 9. Raytracing model with (top) and without (bottom) vacuum chamber
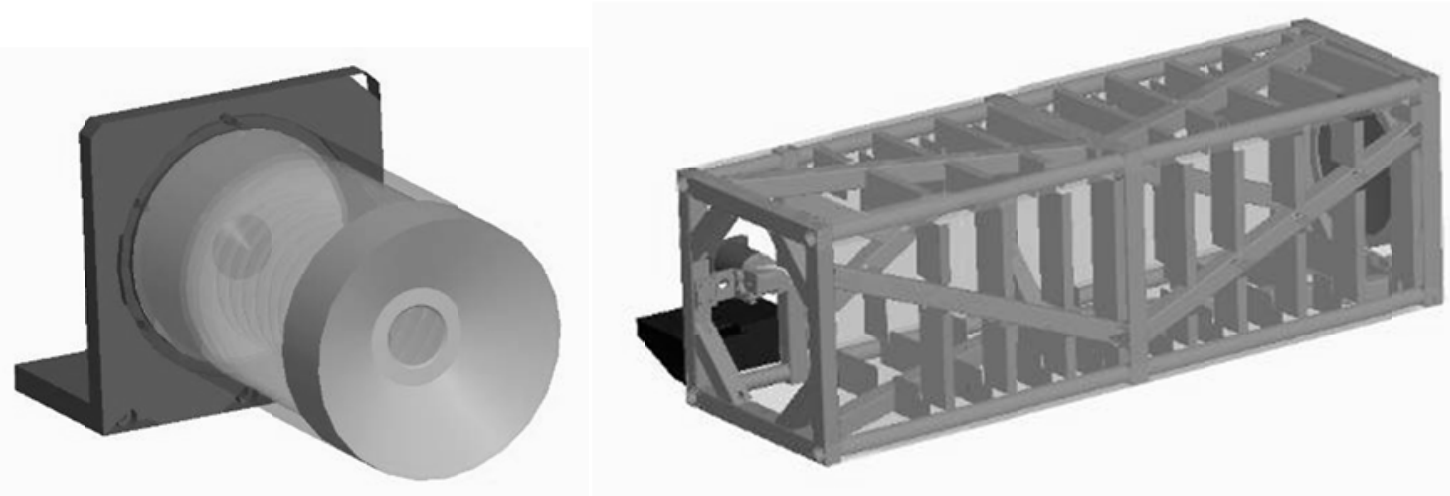

Figure 10. Collimator FPA assembly model (left) and collimator model (right)

All surfaces are black coated with MAP PU1 modeled by a typical black paint with $6 \%$ TIS in normal incidence. 


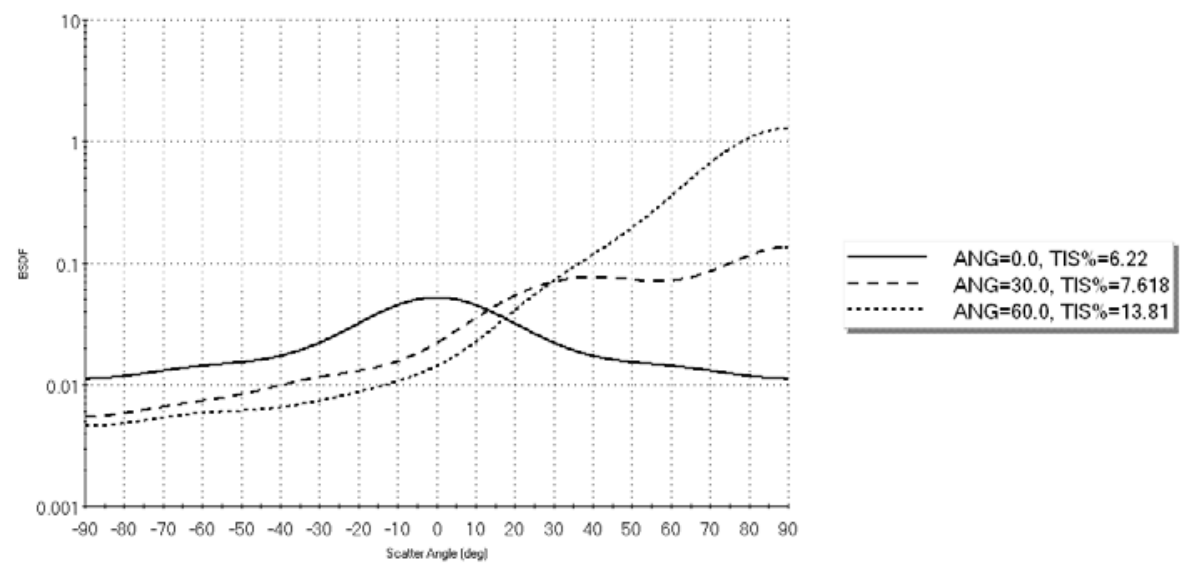

Figure 11. MAP PU1 paint BRDF profiles

\subsection{Strayligth simulation: Nearfield performances}

The nearfield contributions are mainly:

- Straylight induced by optical surfaces scattering (particulate contamination, microroughness contribution, dig contribution);

- Straylight induced by the source assembly;

- Straylight induced by backscattering between the payload baffle and the parabolic mirror.

The optical surface particulate contamination impact is based on MIE scattering BRDF with typically CL100 and CL200 level according MIL-1246C. The CL100 surface cleanliness is mandatory to fit with requirements.

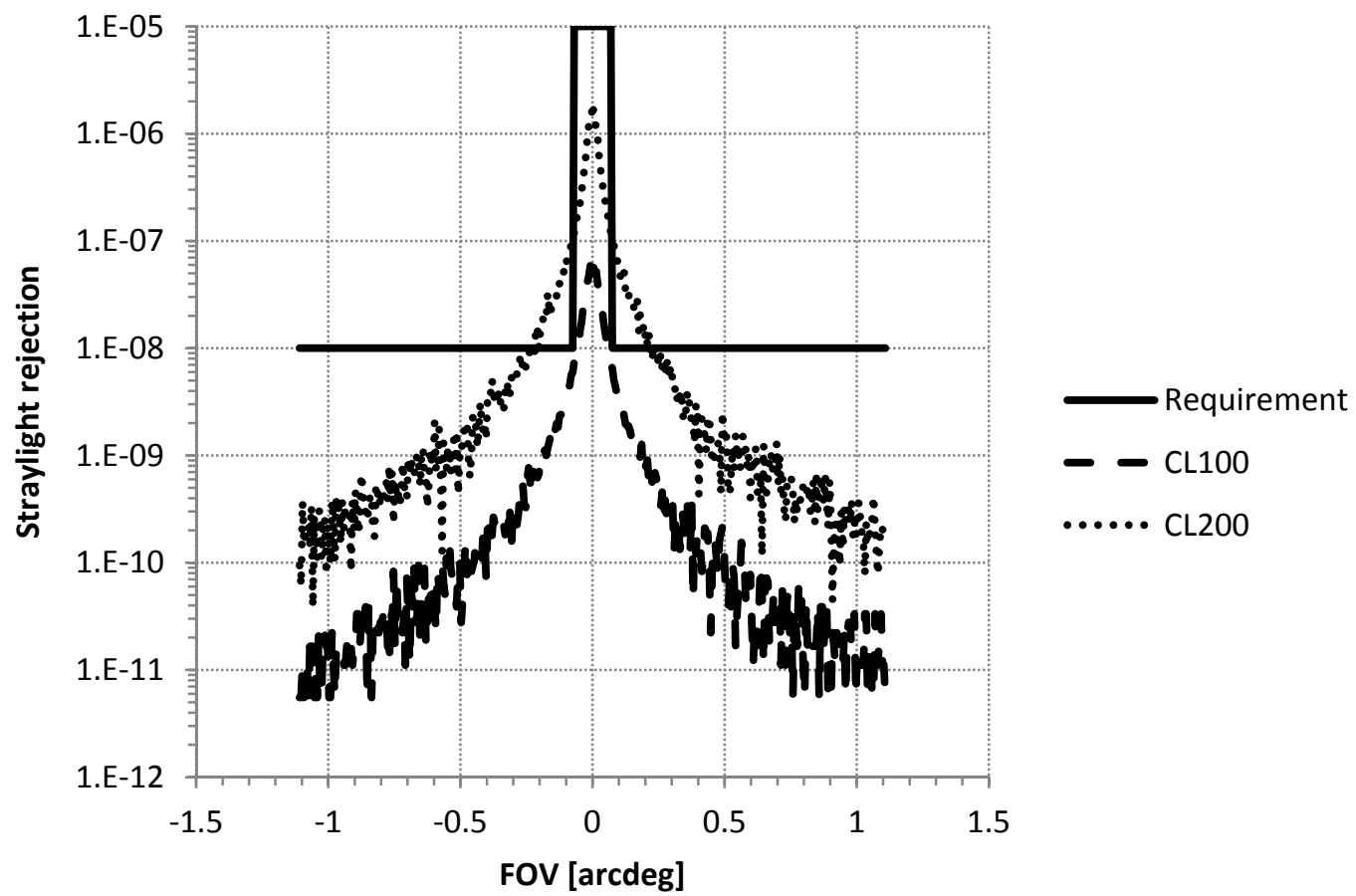

Figure 12. Impact of optical surfaces cleanliness on nearfield straylight. 
The optical surface microroughness BRDF is based on Harvey function with typical parameters. Microroughness contributions is driven by the parabolic mirror microroughness which was not specified for this purposes. As shown in Figure 13, the impact of the microroughness straylight contribution is into requirements.

Table 1: Characteristics and Harvey parameters for collimator mirrors.

\begin{tabular}{|c|c|c|}
\cline { 2 - 3 } \multicolumn{1}{c|}{} & Parabolic mirror & Fold mirror \\
\hline Microroughness (RMS) & $1.2 \mathrm{~nm}$ & $0.4 \mathrm{~nm}$ \\
\hline TIS@805nm & $0.035 \%$ & $0.0038 \%$ \\
\hline Harvey function & $\mathrm{b}_{0}=0.073$ & $\mathrm{~b}_{0}=0.0082$ \\
parameters & $1=0.01$ & $1=0.01$ \\
& $\mathrm{~s}=-1.8$ & $\mathrm{~s}=-1.8$ \\
\hline
\end{tabular}

The scratch and dig of the optical surfaces contribution is mainly driven by the parabolic mirror digs: optical surface is degraded by non negligible digs density $\left(\mathrm{N}=5 \mathrm{~cm}^{-2}\right)$. The digs induced BRDF is based on lambertian and specular contributions ([8]):

$$
\begin{aligned}
& \operatorname{BRDF}(\sin \theta, \phi, N, \lambda)=\frac{1}{4} N \phi^{2}\left[1+\frac{\pi^{2} \phi^{2}}{4 \lambda^{2}}\left(1+\frac{\sin \theta^{2}}{L d^{2}}\right)^{\frac{-3}{2}}\right] \\
& L d=\left(\frac{4}{\pi^{4}}\right)^{\frac{1}{3}} \frac{\lambda}{\phi}
\end{aligned}
$$

Where $\lambda$ is the wavelength, $\Phi$ is the dig diameter and $\mathrm{N}$ is the dig density.

The impact of the optical surfaces digs straylight contribution is above the requirements as shown in Figure 13.

The collimator source assembly straylight contribution is mainly driven by the microroughness on the pyramid. The straylight contribution is negligible.

For nearfield measurements, the payload baffle is implicated in association with the diffusion onto the parabolic mirror (mainly the diffusion from the digs). As shown in Figure 13, this straylight contribution is one order of magnitude below the parabolic mirror dig diffusion.

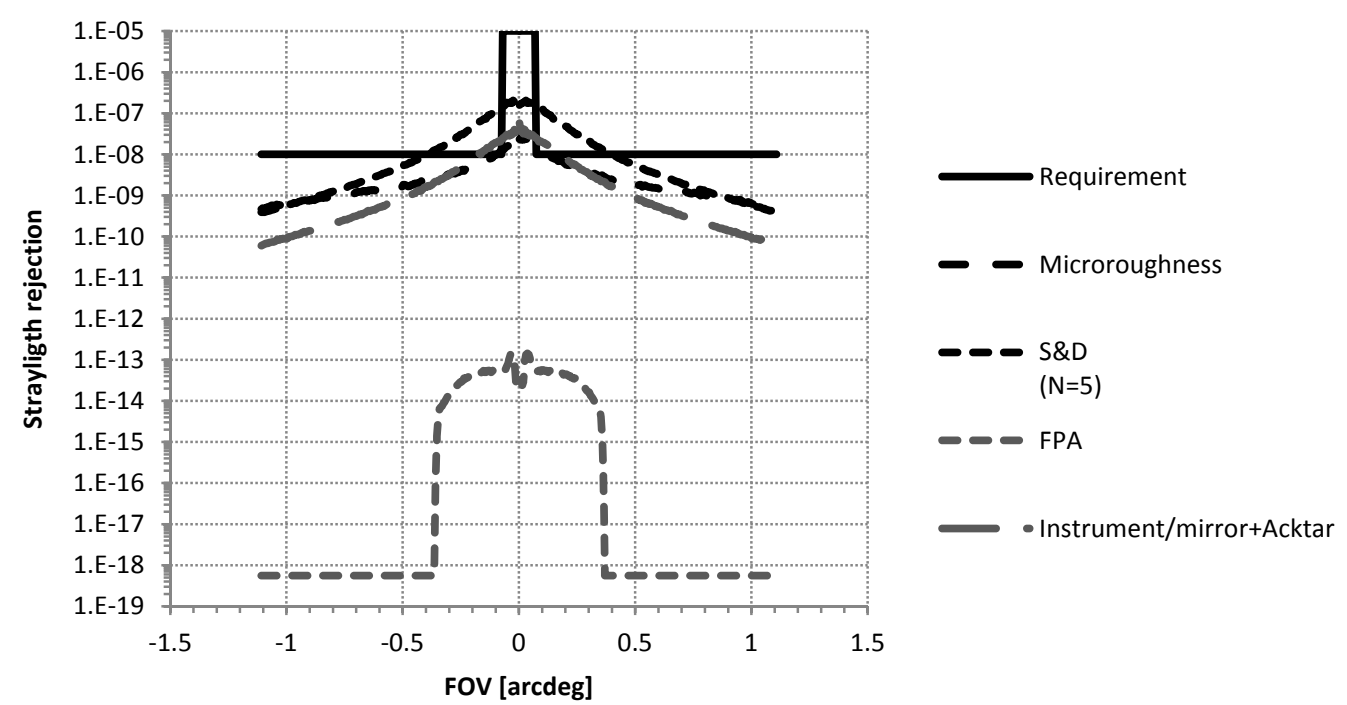

Figure 13. Impact of optical surfaces microroughness and digs and impact of FPA assembly and payload baffle on nearfield straylight. 
Table 2 summarizes the nearfield performances at 4.1 arcmin from the nominal field of view. Excepted the dig straylight contribution, all other contributions fit with the requirement $\left(<10^{-8}\right)$.

Table 2: Straylight contribution for nearfield measurements at FOV $=4.1 \operatorname{arcmin}(250 \operatorname{arcsec})$.

\begin{tabular}{|c|c|}
\hline Optical surfaces microroughness: & $1.24710^{-8} \pm 11 \% @ 1 \sigma$ \\
\hline Optical surface cleanliness (CL100): & $7.5510^{-9} \pm 7 \% @ 1 \sigma$ \\
\hline Parabolic mirror digs (N=5 cm ${ }^{-2}$ ) & $1.5210^{-7} \pm 7 \% @ 1 \sigma$ \\
\hline Source assembly: & $5.510^{-14} \pm 28 \% @ 1 \sigma$ \\
\hline Payload baffle: & $2.4810^{-8}$ \\
\hline Requirements: & $10^{-8}$ \\
\hline
\end{tabular}

\subsection{Straylight simulation: Farfield performances}

The farfield contributions are mainly:

- Straylight induced backscattering between the payload baffle and the black tent;

- Straylight induced backscattering between the payload baffle and the parabolic mirror;

- Straylight induced by the air dust.

The strayligth induced by the black tent is lower than $10^{-12}$ with MAP PU1 and one order of magnitude below by using Acktar ${ }^{\circledR}$ black coating on the payload baffle (Figure 14). The straylight induced by the air dust is limited or suppressed by using a small vacuum into the chamber.

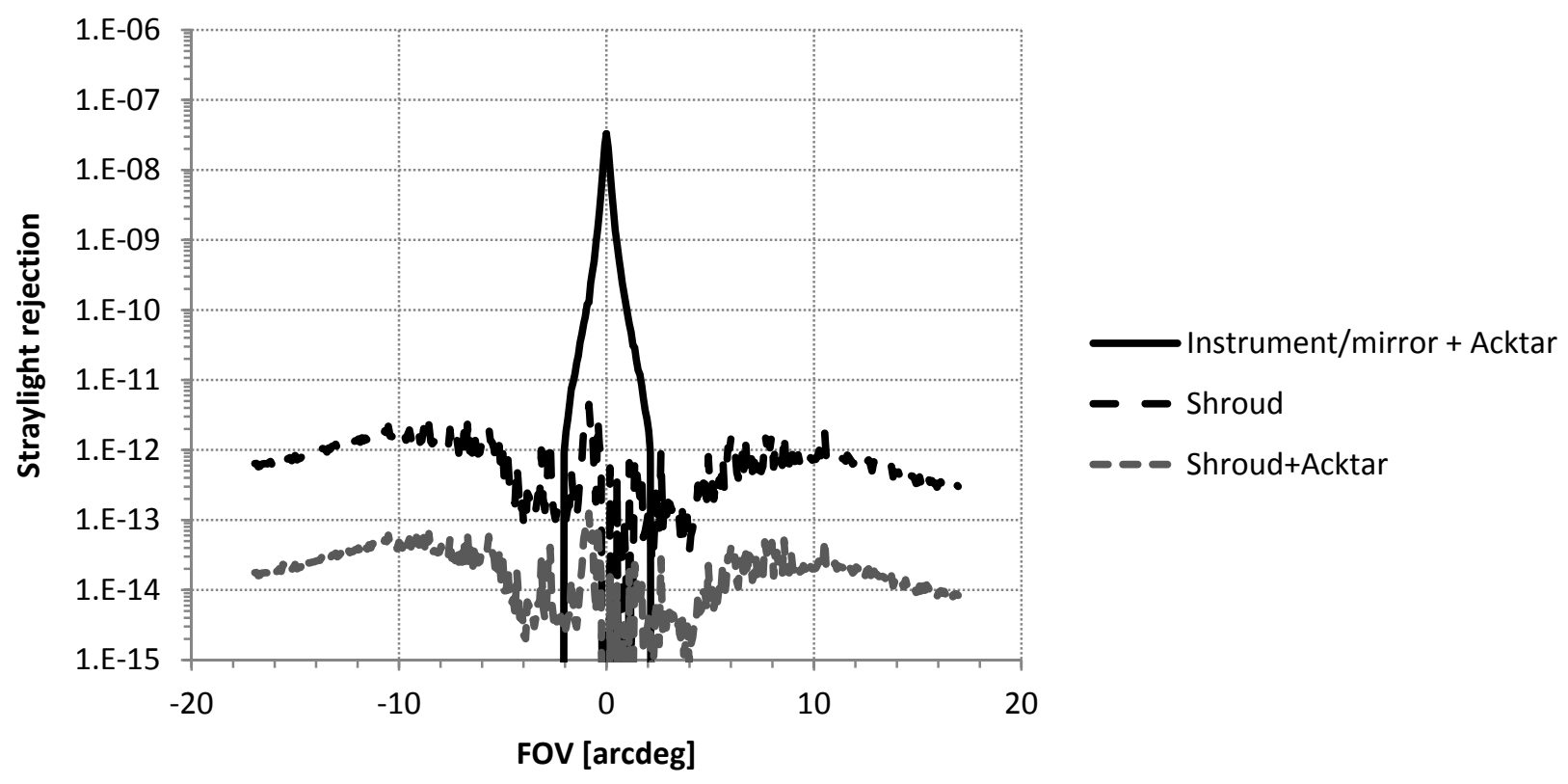

Figure 14. Impact of payload baffle scattering on farfield strayligth. 


\section{FACILITY STATUS}

The facility is building up:

- Collimator source assembly is at black coating level;

- $\quad$ Light source, baffling, black tent, MGSE, monitoring and collimator are available at CSL.

Straylight test on PROBAV QM are foreseen in December 2012. Straylight test on SOLOHI are foreseen in January 2013.

\section{CONCLUSIONS}

Stray light characterization of earth Observation satellites has become a growing necessity to guarantee the mission success. To fulfill this, a new stray light test facility is under development at CSL. In this paper we have demonstrated that the ability to measure PSFs at below $1 \mathrm{E}^{-10}$ should be possible from the visible till the NIR. The facility will be able to test payload of several 100 of $\mathrm{kg}$ with FOV of $+/-300$ arc degrees across track and $+/-15^{\circ}$ along track. The acquire data will not allow to identify each the stray light source mechanisms; it provides the integrated PSF taking into account all the contributors. Nevertheless the big advantage of this stray light test facility is its ability to measure nearfield stray light. The measurement will not only be used for the final acceptance of the instruments but also for removing the stray light contribution from the in flight data images. Simulations gives confidence on the facility performances.

\section{ACKNOWLEGMENTS}

This development would not have been possible without the help of the Belgium GSTP program.

\section{REFERENCE}

[1] Y. Stockman, I. Domken, H. Hansen, JP. Tock, T.A. Decker, A. Rasmussen, A.F.J. den Boggende, J.W. den G-Herder, F. Paerels, D. de Chambure, P. Gondoin, "XMM Flight Modules with Reflection Grating Assembly and X-ray baffle testing”, Proc. SPIE 3444, (1998).

[2] J.Y. Plesseria, E. Mazy, J.M. Defise, P. Rochus, E. Lemmens, D. Vrancken, "Optical and mechanical design of a straylight rejection baffle for COROT", Proc. SPIE 5170, (2003)

[3] JY.Plesseria, E. Mazy, JM. Defise, P. Rochus, A. Magnan, V. Costes, "Straylight analyses of the external baffle of COROT", International Conference on Space Optics - ICSO, (2000)

[4] J.M. Defise, J.P. Halain, E. Mazy, P. Rochus, R.A. Howard, J.D. Moses, D.G. Socker, R. Harrison, G.M. Simnett, "Design and Tests for the Heliospheric Imager of the STEREO Mission”, Proc SPIE 4853, (2003)

[5] E. Mazy, J.P. Halain, J.M. Defise, P. Rochus, R.A. Howard, J.D. Moses, C. Eyles, R. Harrison, "Design and Performances of the Heliospheric Imager for the STEREO Mission", Proc. SPIE 5962, (2005)

[6] A. Zuccaro Marchi, M. Taccola, Jorg Versluys, Didier Beghuin, Yvan Stockman, Ronald Kassel, M. François, Ignacio Torralba Elipe, "PROBA V Multispectral Imager: Status", International Conference on Space Optics - ICSO, (2012)

[7] Yvan Stockman, ML Hellin, A. Zuccaro Marchi, M. Taccola, Jorg Versluys, M. François, E. Mazy, "Conceptual design of a straylight facility for earth observation satellites" International Conference on Space Optics - ICSO, (2012)

[8] G.L. Peterson, "Strayligth analysis with ASAP”, Breault Research Organization, (2010) 